\section{Trial watch}

IMPROVING THE TOLERANCE OF DOSEDENSE CHEMOTHERAPY

Maintaining dose-dense chemotherapy is often hindered by various adverse events, and treatment delay risks reducing treatment efficacy. One such adverse event is severe fatigue, and tumour necrosis factor- $\alpha$ (TNF $\alpha$ ) is one of the cytokines responsible for this. So, J. Paul Monk and colleagues investigated whether a TNF $\alpha$ decoy receptor, etanercept, would enable the maintenance of dose-dense docetaxel in patients with advanced tumours.

Initially, 12 patients were randomized to either docetaxel alone at a dose of $43 \mathrm{mg} \mathrm{m}^{-3}$ a week (cohort A) or the same dose of docetaxel plus $25 \mathrm{mg}$ of etanercept subcutaneously twice a week (cohort B). Patients were asked to record their levels of fatigue on a weekly basis.

More doses of docetaxel ( 35 of 36 doses) were given to patients in cohort $B$, than to patients in cohort A ( 29 of 36 doses; $P=0.055$ ), and, owing to the absence of disease progression or severe toxicity, 3 of the cohort $B$ patients were able to receive additional cycles of treatment. In another set of patients the weekly dose of docetaxel was increased to $52 \mathrm{mg} \mathrm{m}^{-3}$ with etanercept, and neutropaenia rather than fatigue was the dose-limiting toxicity.

Patients who received docetaxel and etanercept self reported significantly less fatigue $(P<0.001)$, and biochemical analyses indicate that etanercept does not affect the pharmacokinetics of docetaxel. Tumour responses to docetaxel were seen only in the patients who received etanercept.

So, adding a TNF $\alpha$ inhibitor to dose-dense chemotherapy regimens improved patient tolerance of the treatment and helped to preserve the dose intensity.

ORIGINAL RESEARCH PAPER Monk, J. P. et al. Assessment of tumour necrosis factor $\alpha$ blockade as an intervention to improve tolerability of dose-intensive chemotherapy in cancer patients. J. Clin. Oncol. 24 1852-1859 (2006)

\section{TESTING FOR EARLY BREAST CANCER}

Biomarker Technologies, a breast cancer diagnostic company in the United States, has received approval from the Western Institutional Review Board to begin a clinical trial on the early detection of breast cancer using a blood test.

The blood test evaluates the levels of expression of 5 biomarkers - prostate-specific antigen, interleukin 6 , interleukin 8 , vascular endothelial growth factor and tumour necrosis factor- $\alpha$. These biomarkers, when combined and adjusted for age, have more than $95 \%$ sensitivity and specificity, and an accuracy of more than $88 \%$ in identifying women with non-treated breast cancer, according to the company's web site.

The trial aims to recruit more than 930 women, and the study is expected to take 4 to 6 months to complete.

The company hopes that this, the first molecular test for breast cancer, will prove more widely applicable than mammography, particularly for women in their 20 s and 30 s.

\title{
Less is more
}

Acute lymphoblastic leukaemia (ALL) that is positive for the translocation fusion product $\mathrm{BCR}-\mathrm{ABL}$ $(\mathrm{Ph}+\mathrm{ALL})$ does not respond well to imatinib therapy. At least $30 \%$ of $\mathrm{Ph}+\mathrm{ALL}$ patients have deletions in cyclin-dependent kinase inhibitor $2 \mathrm{~A}$ (CDKN2A), which encodes the 2 tumour-suppressor proteins INK4a and ARF. Richard Williams and colleagues now show that loss of $\mathrm{ARF}$ is necessary to trigger lympholeukaemias in pre-B cells that express $B c r-A b l$, and that ALLs that arise in mice receiving $\mathrm{Arf} f^{/-} \mathrm{Bcr}-\mathrm{Abl}^{+/+}$pre-B cells do not respond to imatinib.

The authors first introduced $B c r-A b l$, which was tagged with green fluorescent protein or a control vector, into pre-B cells derived from $\mathrm{Arf}^{+/+}, \mathrm{Arf}^{+/-}$or $\mathrm{Arf} \mathrm{f}^{-/-}$mice and cultured them on bone-marrow-derived stromal cells, which produced the lymphopoiesis inducer interleukin 7 (IL-7). Although $\mathrm{Bcr}-\mathrm{Abl}$ eliminates the dependency of pre-B cells on IL-7, induction of Arf by Bcr-Abl triggers p53-dependent apoptosis. However, $\mathrm{Arf}^{-/-}$cells did not die, and continued to proliferate.

So what effect does the loss of Arf have on the development of leukaemia? When $\mathrm{Arf}^{+/+}$bone-marrow progenitors that were transduced with $B c r-A b l$ were introduced into lethally irradiated mice, lympholeukaemias developed slowly, but Arf loss significantly decreased disease latency and increased the clinical severity of the leukaemia. In a second, more stringent ALL model, transiently cultured pre-B cells that expressed $\mathrm{Bcr}-\mathrm{Abl}$ were injected into immunocompetent mice $-\mathrm{Arf}^{+/+}$pre-B cells rarely induced tumours, $A r f^{+/-}$cells induced tumours in which the wild-type Arf allele was inactivated and $A r f^{-/-}$cells rapidly induced highly aggressive, disseminated disease.

As patients with $\mathrm{Ph}+\mathrm{ALL}$ are insensitive to imatinib treatment, the authors investigated whether immunocompetent mice that received $\mathrm{Bcr}-\mathrm{Abl}^{+/+} \mathrm{Arf} f^{\prime-}$ pre-B cells would also prove to be resistant to this drug. Although imatinib inhibited the tyrosine phosphorylation of BCR-ABL, the $A r f^{-/-}$or $A r f^{+/-}$mice developed lympholeukaemia and rapidly died, even if they were maintained on clinically effective doses of imatinib. Importantly, tumour cells that were recovered from moribund mice showed the same sensitivity to imatinib as the donor cells, which implies that resistance was non-cellautonomous. The addition of IL-7 to $B c r-A b l^{+/+} A r f^{-1}$ pre-B cells heightened resistance to imatinib, and the addition of a small-molecule inhibitor of Jak kinases - which inhibits IL-7 signalling - resensitized the cells to imatinib.

The mechanisms by which ARF inactivation overrides sensitivity to imatinib in vivo still need to be elucidated, but the authors reason that a combination of imatinib and a Jak-kinase inhibitor to treat $\mathrm{Ph}+\mathrm{ALL}$ is worth further investigation.

\section{Ezzie Hutchinson}

ORIGINAL RESEARCH PAPER Williams, R. T. et al. Arf gene loss enhances oncogenicity and limits imatinib response in mouse models of $\mathrm{Bcr}-\mathrm{Abl}$ induced acute lymphoblastic leukemia. Proc. Natl Acad. Sci. USA 103, 6688-6693 (2006)

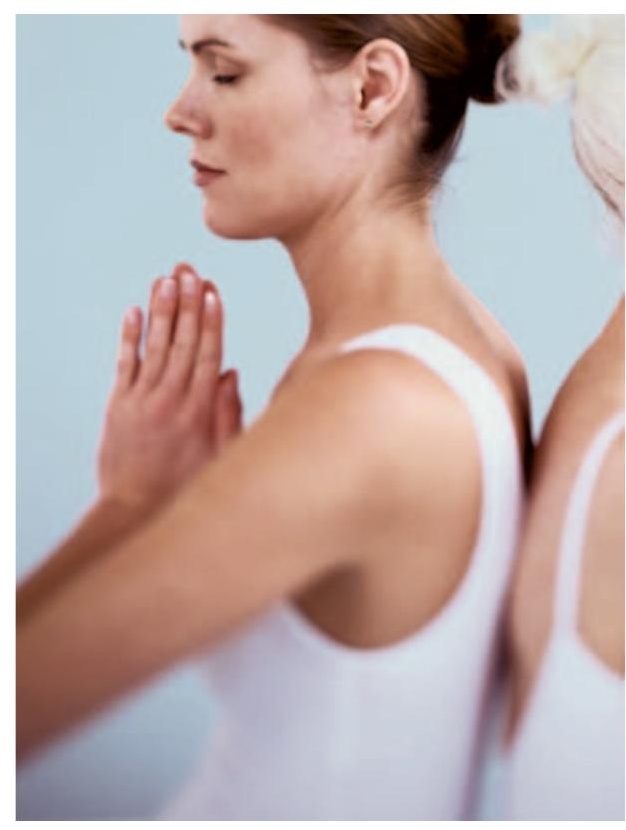

